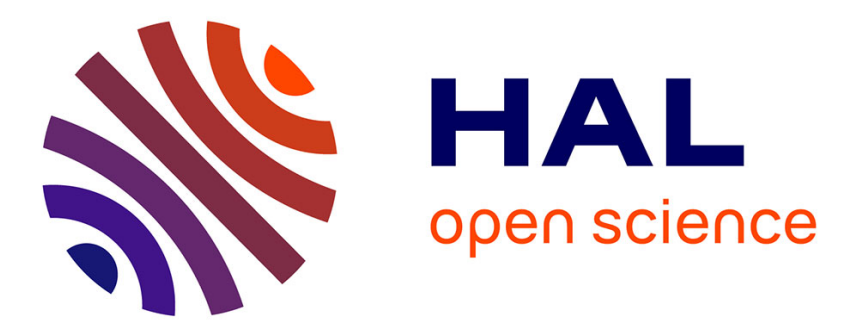

\title{
Low-complexity MMSE turbo equalization : a possible solution for EDGE
}

Christophe Laot, Raphaël Le Bidan, Dominique Leroux

\section{To cite this version:}

Christophe Laot, Raphaël Le Bidan, Dominique Leroux. Low-complexity MMSE turbo equalization: a possible solution for EDGE. IEEE Transactions on Wireless Communications, 2005, 4 (3), pp.965 974. 10.1109/TWC.2005.847095 . hal-01870549

\section{HAL Id: hal-01870549 \\ https://hal.science/hal-01870549}

Submitted on 10 Jun 2021

HAL is a multi-disciplinary open access archive for the deposit and dissemination of scientific research documents, whether they are published or not. The documents may come from teaching and research institutions in France or abroad, or from public or private research centers.
L'archive ouverte pluridisciplinaire HAL, est destinée au dépôt et à la diffusion de documents scientifiques de niveau recherche, publiés ou non, émanant des établissements d'enseignement et de recherche français ou étrangers, des laboratoires publics ou privés. 


\title{
Low Complexity MMSE Turbo Equalization:
}

\section{A Possible Solution for EDGE}

\author{
C. Laot, R. Le Bidan and D. Leroux \\ ENST Bretagne, BP 832, 29285 Brest Cedex, FRANCE \\ Phone: 332290010 14, Fax: 33229001012 \\ E.mail : christophe.laot@enst-bretagne.fr (contact author),
}

raphael.lebidan@enst-bretagne.fr, dominique.leroux@enst-bretagne.fr

\begin{abstract}
This paper deals with a low complexity receiver scheme where equalization and channel decoding are jointly optimized in an iterative process. We derive the theoretical transfer function of the infinite length linear minimum mean square error (MMSE) equalizer with a priori information. A practical implementation is exposed which employs the Fast Fourier Transform (FFT) to compute the equalizer coefficients, resulting in a low complexity receiver structure. The performance of the proposed scheme is investigated for the Enhanced General Packet Radio Service (EGPRS) radio link. Simulation results show that significant power gains may be achieved with only a few (3-4) iterations. These results demonstrate that MMSE turbo equalization is an attractive candidate for singlecarrier broadband wireless transmissions in long delay-spread environments.
\end{abstract}

Indexing terms: mobile communications, fading channel, turbo equalization, iterative decoding, intersymbol interference 


\section{INTRODUCTION}

Providing multimedia access capabilities to mobile terminals requires low-complexity signal processing algorithms to combat fading and intersymbol interference (ISI) which arise in the context of multipath propagation, while trying to minimize the power requirements (and thus the cost) of the reception unit. Turbo equalization [1] combines equalization and channel decoding in a jointly optimized scheme and provides a reliable solution to improve the radio link performance of single-carrier transmissions in severe frequency-selective environments.

The optimal realization of the turbo equalizer relies on a Maximum A Posteriori (MAP) equalizer to combat ISI [2]. In spite of its excellent performance, its use is essentially restricted to short delay-spread channels and low-order modulations (typically binary phase-shift keying BPSK and quaternary phase-shift keying QPSK) for complexity issues. Therefore, alternative equalization strategies have been proposed in the literature. Among them, the class of sub-optimum reduced-state trellis-based equalizers appears as a promising candidate for use in a turbo equalization scheme [3-8]. However, they generally require the use of a front-end prefilter in order to concentrate the energy in the first taps of the equivalent minimum-phase channel to achieve satisfactory performance. Moreover, with the exception of [6], they do not strictly address the increasing complexity issue arising from the use of higher-order modulations. Turbo equalization relying on linear filtering operations [9-16] thus offers a reliable alternative able to deal with multilevel modulation schemes at a relatively low computational cost.

Considering current mobile digital cellular systems such as Global System for Mobile Communications (GSM) in Europe or Industry Standard (IS) 136 in the USA, linear equalization (LE) has been discarded in favor of maximum likelihood sequence detection (MLSD) because of the resulting noise enhancement. However, when used in a turbo equalization scheme, the performance achieved by LE with a priori information 
compares favorably with MAP equalization [11] while retaining a significantly lower complexity.

In this paper, we derive the infinite-length linear Minimum Mean Square Error (MMSE) linear equalizer with a priori information which may be viewed as a particular instance of the maximum Signal to Noise Ratio (SNR) equalizer. By determining the theoretical transfer function of the infinite-length MMSE equalizer, this paper completes publication [10] where the equalizer was realized entirely in adaptive form without paying particular attention to the exchange of probabilistic information between the equalizer and the decoder. In addition, we expose a sub-optimal finite-length realization of this equalizer, where the filter coefficients are computed from preliminary channel estimates using the Fast Fourier Transform (FFT) algorithm. Finally, the performance of the proposed receiver is investigated for various code rates in the context of the Enhanced General Packet Radio Service (EGPRS) standard [3].

The paper is organized as follows. Section II describes the turbo equalization model, where the information data bits are coded, interleaved and then transmitted over a frequency selective channel, using $M$-ary phase-shift keying ( $M$-PSK) or $M$-ary quadrature amplitude-modulated ( $M$-QAM) signaling. The optimum infinite-length MMSE linear equalizer with a priori information is derived in section III in the turbo equalization context. In section IV, we describe a low-complexity computation procedure relying on the FFT to realize the equalizer in finite-length form. Application scenarios for EGPRS radio links are investigated in section V. Conclusions are given in section VI. 


\section{DESCRIPTION OF THE TURBO EQUALIZATION SCHEME}

\section{A. Transmission scheme}

Let us consider the transmission scheme depicted in figure 1. A rate $R$ convolutional encoder (punctured or not) is fed by independent binary data $\alpha_{n}$ taking values in $\{0,1\}$ with equal probability. A random interleaver $\Pi$ shuffles the resulting coded data sequence. Each set of $m$ successive interleaved binary coded bits $c_{n}^{i} ; i=1, \cdots, m$ is mapped onto an $M$-ary complex symbol $d_{n}=f\left(c_{n}^{1}, \cdots, c_{n}^{m}\right)$ with variance $\sigma_{d}^{2}$ at the symbol rate $1 / T$. We restrict ourselves to a Gray labeling map $f($.$) in this work. For M$ QAM signals, the real and imaginary parts of symbol $d_{n}$ take values in the set $\{ \pm 1, \pm 3, \ldots, \pm(\sqrt{M}-1)\}$ with $M=2^{m}$

<Fig. 1>

The signal at the output of the discrete-time equivalent channel is corrupted by additive, zero-mean, circularly symmetric white Gaussian noise $w_{n}$ with total variance $\sigma_{w}^{2}$. The receiver observes the noisy channel output $r_{n}$

$$
r_{n}=\sum_{l=0}^{L} h_{l} d_{n-l}+w_{n}
$$

where the set $\left\{h_{l}\right\}$ denotes the $L+1$ coefficients of the discrete-time equivalent channel model. The transfer function of the channel is given by

$$
\tilde{H}(v)=\sum_{l=0}^{L} h_{l} e^{-j 2 \pi v l T}
$$

with

$$
h_{l}=T \int \tilde{H}(v) e^{j 2 \pi v l T} d v
$$

and where integration is carried out over the Nyquist band $[-1 / 2 T ; 1 / 2 T]$ in the previous expression. 
The signal to noise ratio (SNR) at the turbo equalizer input is given by

$$
\mathrm{SNR}=\frac{\sigma_{d}^{2}}{\sigma_{w}^{2}} E\left\{h h_{0}\right\}=R \frac{\bar{E}_{b}}{N_{0}} \log _{2}(M)
$$

where

$\bar{E}_{b}$ is the average received energy per information bit;

$N_{0}$ is the one-sided noise power spectral density at the receiver input;

$\left\{h h_{l}\right\}$ denotes the discrete-time autocorrelation function of the channel impulse response, defined by

$$
h h_{l}=\sum_{i=-\infty}^{\infty} h_{i} h_{i-l}^{*}
$$

\section{B. Turbo equalization principle}

Following the turbo principle [17][18] as applied to the decoding of serially concatenated turbo codes [19], the turbo equalizer is an iterative receiver where the equalization and decoding operations are repeated several times on the same set of received channel symbols. Each equalization and decoding step is carried out by a module supplied with the channel observations $r_{n}$ as well as with a priori information on the coded bits in Log-Likelihood Ratio (LLR) form delivered by the previous module. We will now describe the structure of a turbo-equalization module.

As shown in figure 2, a soft-input soft-output (SISO) symbol mapper first computes soft estimates $\bar{d}_{n}$ about the transmitted symbol $d_{n}$ from the a priori LLRs available at the input. Together with the channel observation, the resulting symbol estimates enter a linear equalizer. At the equalizer output, a memoryless SISO symbol demapper delivers a sequence of extrinsic information $\mathbf{L}_{e}^{e q}=\left\{L_{e}^{e q}\left(c_{n}^{i}\right)\right\}$ that is deinterleaved and submitted as a priori information, denoted $\mathbf{L}_{a}^{d e c}=\left\{L_{a}^{d e c}\left(c_{n}^{i}\right)\right\}$, to the channel decoder input. Note that, as shown figure 2, the SISO demapper should exploit the a priori information in order to improve performance when non-binary signal sets are considered, especially 
for labeling rules other than Gray mapping [14][21]. The cascade of the SISO mapper, MMSE inner equalizer and SISO demapper forms an overall SISO MMSE equalizer. On the basis of the extrinsic LLRs delivered by the demapper, the SISO channel decoder implements the Log-MAP algorithm [20] and computes a posteriori LLRs on the coded bits

$$
L^{d e c}\left(c_{k}^{i}\right)=\log \frac{\operatorname{Pr}\left\{c_{k}^{i}=1 / \mathbf{L}_{a}^{d e c}\right\}}{\operatorname{Pr}\left\{c_{k}^{i}=0 / \mathbf{L}_{a}^{d e c}\right\}}
$$

These a posteriori LLRs $\mathbf{L}^{\text {dec }}=\left\{L^{\text {dec }}\left(c_{n}^{i}\right)\right\}$ are interleaved again and delivered to the next module as updated a priori information $\mathbf{L}_{a}^{e q}=\left\{L_{a}^{e q}\left(c_{n}^{i}\right)\right\}$ for a new equalization and decoding step. This soft information exchange allows the equalizer to benefit from the coding gain and vice-versa.

Note that in contrast with common practice, we purposely feed back here the entire $a$ posteriori information from the decoder to the equalizer. When considering MMSE turbo-equalization of high-order modulation, we have noticed non negligible performance degradations when feeding back only the extrinsic information. Interestingly, other researchers have come up with the same observation in a similar context [22].

<Fig. 2>

\section{DESCRIPTION OF THE SISO MMSE EQUALIZER}

\section{A. SISO mapper}

The soft estimates $\bar{d}_{n}$ of $d_{n}$ are computed from the knowledge of the a priori LLRs provided by the channel decoder at the previous iteration. We retain the conditional mean estimator [23] defined by

$$
\bar{d}_{n}=E\left\{d_{n} / \mathbf{L}_{a}^{d e c}\right\}
$$


where $\mathbf{L}_{a}^{\text {dec }}$ denotes the vector of a priori LLRs feeding the SISO decoder. Under the assumption of independent modulated bits owing to the presence of the bit interleaver, we have

$$
\bar{d}_{n}=\sum_{d=f\left(c^{1}, \cdots, c^{m}\right) \in \mathrm{D}} d \prod_{i=1}^{m} \operatorname{Pr}\left\{c_{n}^{i}=c^{i} / \mathbf{L}_{a}^{d e c}\right\}
$$

where the summation is carried out over the $M$-ary complex symbols $d$ in the signal set $D$, and where the coded bits $c^{i}$ take the value 0 or 1 according to the considered symbol $d$ and the labeling rule $f\left(c^{1}, \cdots, c^{m}\right)$. By taking into account the correspondence between $L^{\text {dec }}\left(c_{k}^{i}\right)$ and $L_{a}^{e q}\left(c_{n}^{i}\right)$, the conditional probability $\operatorname{Pr}\left\{c_{n}^{i}=c^{i} / \mathbf{L}_{a}^{d e c}\right\}$ may be obtained from (6) by

$$
\operatorname{Pr}\left\{c_{n}^{i}=c^{i} / \mathbf{L}_{a}^{d e c}\right\}=\frac{1}{2}\left(1+\left(2 c^{i}-1\right) \tanh \left(\frac{L_{a}^{e q}\left(c_{n}^{i}\right)}{2}\right)\right), \quad c^{i}=0,1
$$

The latter expression shows that although the soft symbol estimates $\bar{d}_{n}$ are defined with respect to the a priori information $\mathbf{L}_{a}^{\text {dec }}$ feeding the decoder input in (7), they are computed using the a posteriori LLRs delivered by the decoder.

It is easily shown that the soft symbol estimates $\bar{d}_{n}$ have zero mean

$$
E\left\{\bar{d}_{n}\right\}=E\left\{E\left\{d_{n} / \mathbf{L}_{a}^{\text {dec }}\right\}\right\}=E\left\{d_{n}\right\}=0
$$

In addition, we shall see later that the variance $\sigma_{\dot{d}}^{2}$ of the soft symbol estimates $\bar{d}_{n}$ is required in the derivation of the equalizer's transfer function. This quantity may be estimated over a received burst with duration $K$ symbols using the sample mean estimator and property (10), yielding

$$
\sigma_{\bar{d}}^{2}=E\left\{\left|\bar{d}_{n}\right|^{2}\right\} \approx \frac{1}{K} \sum_{n=1}^{K}\left|\bar{d}_{n}\right|^{2}
$$

It can be shown that when $\left|L^{d e c}\left(c_{n}^{i}\right)\right| \rightarrow \infty$ (perfect a priori knowledge), $\bar{d}_{n} \rightarrow d_{n}$ and $\sigma_{\bar{d}}^{2} \rightarrow \sigma_{d}^{2}$. On the other hand, assuming uniform prior knowledge, $\bar{d}_{n} \rightarrow 0$ and $\sigma_{\bar{d}}^{2} \rightarrow 0$. 
These observations justify the use of $\bar{d}_{n}$ as a soft estimate of $d_{n}$. Note furthermore that parameter $\sigma_{\bar{d}}^{2}$ measures the reliability of these estimates.

\section{B. Theoretical infinite-length MMSE equalizer}

In this section, we derive the expression of the infinite-length MMSE linear equalizer in the presence of a priori information about the transmitted symbols, which is referred to as the "ISI Canceller - Linear Equalizer" (IC-LE) in the sequel. Figure 3 depicts the equalizer structure, which consists of two transversal filters operating on the received samples $r_{n}$ and the soft estimates $\bar{d}_{n}$, respectively.

\section{<Fig. 3>}

From (1) and figure 3, the output of the equalizer is given by

$$
s_{n}=\sum_{l} g_{l} d_{n-l}-\sum_{l \neq 0} q_{l} \bar{d}_{n-l}+\sum_{l} p_{l} w_{n-l}
$$

where $\left\{p_{l}\right\}$ and $\left\{q_{l}\right\}$ are the coefficients of the transversal filters with respective transfer functions $\tilde{P}(v)$ and $\tilde{Q}(v)$, and where we have introduced the transfer function $\tilde{G}(v)$ with coefficients $\left\{g_{l}\right\}$ defined by

$$
\tilde{G}(v)=\tilde{P}(v) \tilde{H}(v)
$$

Following the turbo-principle, the output of the equalizer $s_{n}$ should be independent of the a priori symbol $\bar{d}_{n}$ at time $n$. Consequently and as depicted in figure 3 , we impose the condition $q_{0}=0, q_{0}$ being the reference tap of the feedback filter.

From (12), the equalized sample $s_{n}$ at time $n$ can be expressed by

$$
s_{n}=g_{0} d_{n}+\eta_{n}
$$

where $\eta_{n}$ denotes the residual interference and the filtered noise at the equalizer output. We shall assume in the sequel that $\eta_{n}$ follows a complex Gaussian distribution with 
zero-mean and variance $\sigma^{2}$. Although this assumption does not rigorously hold, it simplifies the derivation and gives good results in practice. Then, we can define the signal to noise ratio at the equalizer output as

$$
S N R_{\text {out }}=\left|g_{0}\right|^{2} \sigma_{d}^{2} / \sigma^{2}
$$

The transfer function of the equalizer has been calculated according to the maximization of the quantity $S N R_{\text {out }}$ (see appendix). We obtain the following theoretical transfer functions for the infinite-length IC-LE filters

$$
\begin{gathered}
\tilde{P}(v)=\lambda \frac{\tilde{H}^{*}(v)}{|\tilde{H}(v)|^{2}\left(\sigma_{d}^{2}-\sigma_{\bar{d}}^{2}\right)+\sigma_{w}^{2}} \\
\tilde{Q}(v)=\tilde{H}(v) \tilde{P}(v)-T \int \tilde{H}(v) \tilde{P}(v) d v
\end{gathered}
$$

Different equalizers are obtained depending on the value of the scalar parameter $\lambda$. For example, setting $\lambda=1$ yields the infinite-length unbiased MMSE IC-LE. We consider here the particular case of the minimization of the mean-square error (MSE)

$$
M S E=E\left\{\left|s_{n}-d_{n}\right|^{2}\right\}
$$

We obtain the following value for $\lambda$ (see appendix)

$$
\lambda=\sigma_{d}^{2} /\left(1+\beta \sigma_{a}^{2}\right)
$$

where we have introduced the quantity

$$
\beta=T \int \frac{|\tilde{H}(v)|^{2}}{|\tilde{H}(v)|^{2}\left(\sigma_{d}^{2}-\sigma_{\bar{d}}^{2}\right)+\sigma_{w}^{2}} d v
$$

Interestingly, it turns out that the equalizer performance is completely characterized by the knowledge of the single reference coefficient $g_{0}$. In particular, we note that the mean-square error and the signal to noise ratio at the equalizer output are given by the following expressions (see appendix)

$$
\begin{gathered}
M S E=\left(1-g_{0}\right) \sigma_{d}^{2} \\
S N R_{\text {out }}=\frac{g_{0}}{1-g_{0}}=\frac{\sigma_{d}^{2}-M S E}{M S E} \\
-9-
\end{gathered}
$$


with

$$
g_{0}=\lambda \beta
$$

Using (15) and (22), one easily obtains the variance $\sigma^{2}$ characterizing the Gaussian conditional distribution at the equalizer output

$$
\sigma^{2}=g_{0}\left(1-g_{0}\right) \sigma_{d}^{2}
$$

This parameter is required by the SISO demapper (see section III.C).

One readily verifies from (16)-(20) that for the particular values $\sigma_{\bar{d}}^{2}=0$ and $\sigma_{\bar{d}}^{2}=\sigma_{d}^{2}$, the IC-LE becomes equivalent to a classical MMSE linear equalizer (LE) [24] and to an ideal MMSE interference canceller (IC) [25-26] respectively. Consequently, the IC-LE presents a configurable structure that adapts the equalization strategy according to the reliability of the soft symbol estimates $\bar{d}_{n}$, as measured by the parameter $\sigma_{\bar{d}}^{2}$.

Note finally that the structure of the IC-LE depicted in figure 3 can be modified in order to decrease the overall number of filters coefficients. The resulting configuration is shown in figure 4 and can be identified with the equalization scheme proposed in [1114].

<Fig. 4>

We showed in [27] that under finite-length realization constraints, the IC-LE becomes rigorously equivalent to the approximate MMSE LE (I) proposed by Tüchler et al. in [12, sec. III.B].

\section{SISO demapper}

The SISO demapper [21] computes a posteriori LLRs on the coded bits $L^{e q}\left(c_{n}^{i}\right)$ given the knowledge of the two observations $s_{n}$ and $\mathbf{L}_{a}^{\text {dec }}$ which are the equalizer output and the a priori LLRs feeding the SISO decoder respectively 


$$
L^{e q}\left(d_{n}^{i}\right)=\log \frac{\operatorname{Pr}\left\{c_{n}^{i}=1 / s_{n}, \mathbf{L}_{a}^{d e c}\right\}}{\operatorname{Pr}\left\{c_{n}^{i}=0 / s_{n}, \mathbf{L}_{a}^{d e c}\right\}} \quad i=1, \ldots, m
$$

Applying Bayes rules, (25) becomes

$$
L^{e q}\left(c_{n}^{i}\right)=\log \frac{\sum_{d: c^{i}=1} p\left\{s_{n} / d, \mathbf{L}_{a}^{d e c}\right\} \operatorname{Pr}\left\{d_{n}=d / \mathbf{L}_{a}^{d e c}\right\}}{\sum_{d: c^{i}=0} p\left\{s_{n} / d, \mathbf{L}_{a}^{d e c}\right\} \operatorname{Pr}\left\{d_{n}=d / \mathbf{L}_{a}^{d e c}\right\}} \quad i=1, \ldots, m
$$

where $d: c^{i}=j$ denotes the set of symbols $d=f\left(c^{1}, \cdots, c^{m}\right) \in \mathrm{D}$ whose $i$-th bit $c^{i}$ has value $j=\{0 ; 1\}$.

Using the results of section III.B, the conditional probability $p\left(s_{n} / d, \mathbf{L}_{a}^{\text {dec }}\right)$ is given by

$$
p\left\{s_{n} / d, \mathbf{L}_{a}^{d e c}\right\}=\left(\pi \sigma^{2}\right)^{-1} \exp \left(-\left|s_{n}-g_{0} d\right|^{2} / \sigma^{2}\right)
$$

according to the Gaussian assumption at the equalizer output. In addition, the conditional probabilities on the transmitted symbols are computed from the a priori binary LLRs provided by the decoder as follows

$$
\operatorname{Pr}\left\{d_{n}=d / \mathbf{L}_{a}^{d e c}\right\}=\prod_{p=1}^{m} \operatorname{Pr}\left\{c_{n}^{p}=c^{p} / \mathbf{L}_{a}^{d e c}\right\}
$$

where $\operatorname{Pr}\left\{c_{n}^{p}=c^{p} / \mathbf{L}_{a}^{d e c}\right\}$ is given by (9). The extrinsic information is finally defined as

$$
L_{e}^{e q}\left(c_{n}^{i}\right)=L^{e q}\left(c_{n}^{i}\right)-L_{a}^{e q}\left(c_{n}^{i}\right)
$$

From (6), (26) and noting the correspondence between $L^{d e c}\left(c_{k}^{i}\right)$ and $L_{a}^{e q}\left(c_{n}^{i}\right)$, we obtain the following expression for the extrinsic information at the SISO demapper output

$$
L_{e}^{e q}\left(c_{n}^{i}\right)=\log \frac{\sum_{d: c^{i}=1}\left(p\left(s_{n} / d, \mathbf{L}_{a}^{d e c}\right) \prod_{p=1, p \neq i}^{m} \operatorname{Pr}\left\{c_{n}^{p}=c^{p} / \mathbf{L}_{a}^{d e c}\right\}\right)}{\sum_{d: c^{i}=0}\left(p\left(s_{n} / d, \mathbf{L}_{a}^{d e c}\right) \prod_{p=1, p \neq i}^{m} \operatorname{Pr}\left\{c_{n}^{p}=c^{p} / \mathbf{L}_{a}^{d e c}\right\}\right)}
$$




\section{PRACTICAL REALIZATION OF THE INFINITE-LENGTH EQUALIZER}

We focus in this paper on burst transmissions over quasi-static multipath Rayleigh fading channels where the channel coefficients are assumed to be constant over the burst duration. Hence, the equalizer settings need only be computed once per burst. The optimum transfer function $\tilde{P}(v)$ for the MMSE linear equalizer with a priori information was derived in section III.B in infinite-length form, and thus does not lend itself to a direct implementation with finite impulse response (FIR) filters. Different approaches can be used in practice to obtain the filter coefficients under finite-length realization constraints.

In [9-10], the equalizer coefficients were computed using standard adaptive filtering algorithms with $O(N)$ complexity, where $N$ is the number of filter taps. This method does not require any preliminary channel estimation step. However a long training sequence may be necessary for the equalizer to reach its steady-state, thereby restricting the application of this strategy for long bursts or continuous-mode transmissions over time-invariant or slow-fading channels. Considering now short burst transmissions, the equalizer coefficients are usually computed from an estimate of the channel impulse response, which is in turn typically obtained from known short training sequences embedded into each data block. Calculation of the optimum filter coefficients under finite-length realization constraints then involves a matrix inversion that can be carried out with $O\left(N^{2}\right)$ operations when the channel convolution matrix is Toeplitz. In this context, we showed in [26] that the finite-length IC-LE becomes rigorously equivalent to the approximate MMSE-LE (I) proposed by Tüchler et al. in [12, sec. III.B], so that the time-recursive computation procedures suggested in [11-14] apply as well. In the present paper, we consider instead a computationally efficient (but sub-optimal) alternative method relying on the Fast Fourier Transform to calculate the equalizer coefficients. 
This method involves evaluating the infinite-length transfer function $\tilde{P}(v)$ in $(16)$ at the set of $N$ uniformly-spaced discrete frequencies $\left\{v_{n}=n / N T\right\}$, yielding

$$
\tilde{P}(n / N T)=\lambda \frac{\tilde{H}^{*}(n / N T)}{|\tilde{H}(n / N T)|^{2}\left(\sigma_{d}^{2}-\sigma_{d}^{2}\right)+\sigma_{w}^{2}} \quad n=0, \ldots, N-1
$$

Such a computation can be carried out efficiently in $O\left(N \log _{2} N\right)$ operations with the FFT when $N$ is chosen as a power of 2. Then, Performing an inverse FFT on the sampled frequency-response returns the impulse response $\left\{p_{n}\right\}$ of the equalizer over $N$ points in the time-domain. The impulse response $\left\{q_{n}\right\}$ of the feedback filter $\tilde{Q}(v)$ given by (17) is then finally obtained as the convolution of $\left\{p_{n}\right\}$ with $\left\{h_{n}\right\}$. We emphasize that the proposed computation procedure is inherently sub-optimal with respect to the matrix approach since it technically involves approximating a Toeplitz matrix by a circulant matrix, an assumption that rigorously holds only asymptotically, for $N \rightarrow \infty$. Hence, its accuracy will depend on the number of points considered in the FFT operations. This issue will be examined in greater detail in section V.C.

We have focused so far on the computational cost associated with the determination of the equalizer coefficients. However, the filtering operations are usually more expensive in terms of implementation complexity. We have restricted ourselves to time-domain filtering in this paper. Frequency-domain filtering and more generally frequencydomain equalization may nevertheless be preferable over very long delay-spread channels, as pointed out for example in [27] and [28].

\section{SIMULATION RESULTS}

\section{A. Burst transmission over mobile radio channels}

Simulations of the proposed scheme were conducted in the context of EGPRS that is part of the Enhanced Data rates for GSM Evolution (EDGE) radio access scheme. The 
burst structure is described in [29-30]. A burst includes a middle training sequence of 26 symbols and 8.25 guard symbols at the end. It carries 2x58 payload data symbols, each symbol comprising 3 bits. EGPRS uses 8-PSK modulation combined with a linearized Gaussian minimum-shift keying (GMSK) pulse shaping filter [31-32], allowing 8-PSK to fit into the GSM spectrum mask. We will show in the sequel that this pulse introduces ISI, which makes equalization mandatory at the receiver side, even on the ideal bandlimited additive white Gaussian noise (AWGN) channel. A Gray-labeling map $f($.$) was considered in our simulations. The additional signal set rotation that is$ advocated before transmit filtering in the GSM technical specification [32] was not taken into account, for the sake of simplicity. Note however that the influence of this rotation on the performance is negligible [3].

For the system simulations, we used the following channel models specified in the GSM standard [33]: STatic (ST) (time-invariant channel with no time dispersion), Typical Urban (TU), Hilly Terrain (HT), and EQualizer test (EQ) with 6 taps. For the TU, HT and EQ channels, we considered a slow-varying Rayleigh fading channel (i.e. low mobility). The channel coefficients are then assumed to remain constant during the transmission of a burst. Moreover, we assume ideal frequency hopping (iFH) so that consecutive bursts face independent channel realizations. The receiver filter is a squareroot raised cosine filter with roll-off factor 0.3.

The overall channel model accounting for the linearized GMSK transmit pulse, the mobile radio channel and the receiver filter was computed with an oversampling factor equal to 36. The power distribution of the resulting channel impulse response for the different channel profiles is presented in figure 5 . We observe that the length of the overall impulse response depends on the channel model. After symbol-rate sampling, the discrete-time equivalent channel model spans $L+1=3,4,7$ and 7_symbol periods for the ST, TU, HT and EQ profiles respectively. 


\section{<Fig. 5>}

\section{B. The simulation context for EGPRS}

The EGPRS specifications define 9 coding schemes [34]. Five among them (MCS-5 to MCS-9) employ 8-PSK signaling. For the sake of simplicity, the performance results will be examined only over the user data which are encoded by a rate- $R=1$ / 3 nonrecursive non-systematic convolutional encoder with constraint length 7 and octal generator polynomials $133,171,145$. Different puncturing patterns are used to obtain various code rates. In our simulations, we focused on the MCS-5, MCS-8 and MCS-9 coding schemes with user data code rates $R=0.37, R=0.92$ and $R=1$ respectively. Our implementation closely follows the GSM technical recommendations, with the exception of the deterministic interleaver which has been replaced by a pseudo-random permutation. The coded punctured user data bits combined with the header part and the flag result in a block of 1392 bits. These bits are partitioned over 4 data blocks of 348 bits, i.e. 2x58 8-PSK symbols, which are finally mapped onto 4 different bursts.

The radio link performance is characterized by the block error rate (BLER) measured after decoding versus $\bar{E}_{b} / N_{0}$ (4). A packet (458 information bits) is rejected if at least one information bit is erroneous after decoding. Note that this criterion is more pessimistic than the more practical one based on the use of a Cyclic Redundancy Check (CRC) code. Simulations are stopped when 250 packets have been discarded. The bit error rate (BER) versus $\bar{E}_{b} / N_{0}$ is also evaluated. Our simulations assume both perfect channel estimation and synchronization. Note that the signal to noise ratio $\bar{E}_{b} / N_{0}$ does not take into account the bandwidth expansion caused by the training sequence.

The reference curve, represented by a dashed-dotted line, corresponds to the ideal Matched Filter Bound (MFB). It was obtained experimentally from the IC-LE equalizer supplied with perfect knowledge about the transmitted data symbols (see section III.B). 
Note that for the ST channel, the discrete-time equivalent channel has three timeinvariant coefficients equal to [0.29 0.910 .29$]$ respectively. In this case, the MFB corresponds to the performance of the coded transmission scheme over an ISI-free AWGN channel. Five iterations are performed at the receiver side. They are represented as solid lines on the plots.

\section{Accuracy of the FFT-based coefficient computation procedure}

In this section, we investigate the accuracy of the low-complexity coefficient computation procedure described in section IV according to the number $N$ of FFT points, and compares it with the optimum calculation method involving a matrix inversion. Figure 6 presents the BLER performance of the turbo-equalizer obtained over the TU channel model with the MCS-8 coding scheme at the first iteration. Note that the IC-LE then reduces to a classical MMSE LE. This constitutes a worst-case study since as the reliability of the data estimates increases across the iterations, the IC-LE converges towards an ideal MMSE IC which generally requires fewer coefficients than an MMSE LE. We observe that choosing $N=32$ points for the FFT method provides close to optimum performance in this context, except at high SNR. In the latter case, when the noise variance is small, the MMSE LE approaches a Zero-Forcing LE and more coefficients are then required with the FFT method to compensate for deep fading in the channel frequency response.

\section{<Fig. 6>}

We retained the FFT computation procedure with $N=32$ coefficients for the following simulation results in this section.

\section{Performance comparison between MMSE and MAP turbo equalization}

We define a MAP turbo equalizer and an MMSE turbo equalizer as the iterative receivers employing a MAP equalizer or the MMSE IC-LE of section III respectively. 
Figure 7 presents the simulation results (BLER and BER) obtained over the timeinvariant ST channel model in the MCS-5 context. At signal to noise ratios greater than $3 \mathrm{~dB}$, only 4 iterations are necessary to reach a steady-state of the iterative process which closely approaches the reference (MFB) performance. We thus observe that the intersymbol interference caused by the GSM pulse is almost completely cancelled by the turbo equalizer without noise enhancement at high enough SNR. We also note that MAP turbo equalization outperforms MMSE turbo equalization at low SNR $(<3 \mathrm{~dB})$. However, the performance gain remains marginal with respect to the complexity required by the MAP equalizer (64 states). Finally, we notice a significant performance improvement of several $\mathrm{dB}$ between the first and the fifth iteration, independently of the equalizer considered. This demonstrates the benefits offered by an iterative equalization and decoding process in this context.

\section{<Fig. 7>}

Figure 8 compares the performance obtained with the MAP and MMSE turbo equalization schemes over the time-varying TU channel in the MCS-5 context. At the third iteration, the BLER performance of the MAP turbo-equalizer reaches the MFB bound even at low SNR, whereas we observe a performance loss of about $1 \mathrm{~dB}$ in terms of BER. This phenomenon actually results from the fact that one erroneous block can cause a large number of bit errors and thus severely affect the BER performance. Note also that although the performance of the MMSE turbo equalizer is slightly worse than that of MAP turbo equalization, its complexity is significantly lower. The MMSE ICLE equalizer involves only linear filtering operations whereas the corresponding MAP equalizer operates here on a 512-state trellis.

\section{$<$ Fig. 8>}




\section{E. Performance of the MMSE IC-LE turbo-equalizer over long delay-spread channels}

Figure 9 presents the performance of the MMSE IC-LE turbo equalizer in the EGPRS/MCS-5 context over the HT and EQ time-varying channels. The ability of the MMSE turbo equalizer to exploit the high diversity offered by long delay-spread channels is demonstrated in the EQ channel simulation. We observe in particular that MMSE turbo-equalization achieves significant power gains over the conventional approach where equalization and decoding are performed separately, using only a few (3-4) iterations. In addition, it is worth noting that the performance gain is greater in terms of BLER than in terms of BER, a situation that is particularly attractive for packet transmissions.

\section{<Fig. 9>}

\section{F. Performance of the MMSE IC-LE turbo equalizer as a function of the code rate}

Finally, Figures 10 and 11 present the performance of the MMSE turbo equalizer in EGPRS/MCS-8 and MCS-9 contexts over the time-invariant ST channel and the timevarying TU channel. Again, we observe that the iterative receiver offers remarkable performance gains between the first and the fifth iterations. These results actually demonstrate that turbo-equalization may be beneficial even for transmission scenarios with very high code rates. We recall that the code rate is increased from $1 / 3$ to 1 by proper puncturing in the MCS-9 context.

\section{$<$ Fig. 10>}

$<$ Fig. 11>

\section{CONCLUSION}

This paper describes an infinite-length MMSE turbo equalizer that relies on a lowcomplexity linear equalizer. A computationally efficient coefficient computation procedure relying on the FFT has been described in order to realize the equalizer in 
finite-length form. The resulting receiver appears as a promising candidate for improving the performance of existing single-carrier packet transmission schemes. Simulations of the turbo equalizer in EGPRS / MCS-5, MCS-8 and MCS-9 contexts show that $3 \mathrm{~dB}$ to $5 \mathrm{~dB}$ power gains can be achieved on the BLER performance in comparison with conventional receivers performing one-time equalization and decoding. Moreover, owing to its low complexity, this receiver overcomes the difficulty arising with high-order modulation (8-PSK and above) in comparison with existing reduced-complexity trellis-based equalization algorithms. In particular, the prefilter that is usually required at the receiver front-end in the latter case in order to concentrate the channel energy in the first taps is no-longer necessary. Note finally that the proposed receiver is particularly interesting when the channel exhibits long delay-spreads with high diversity. Low-complexity MMSE turbo-equalization thus appears as an attractive solution for future single-carrier broadband wireless access systems operating in nonline-of-sight (NLOS) environments. 


\section{APPENDIX}

This appendix details the derivation of the MMSE IC-LE transfer function. This demonstration has been inspired by the results presented in [15] by Chan and Wornell.

To maximize the quantity $S N R_{\text {out }}(15)$, the variance $\sigma^{2}$ has to be determined. Using relations (12) and (14), we have

$$
\sigma^{2}=E\left\{\left|s_{n}-g_{0} d_{n}\right|^{2}\right\}
$$

Then, from (12) and (32), it follows that

$$
\sigma^{2}=E\left\{\left|\sum_{l \neq 0} g_{l} d_{n-l}-\sum_{l \neq 0} q_{l} \bar{d}_{n-l}+\sum_{l} p_{l} w_{n-l}\right|^{2}\right\}
$$

The noise samples are independent of the data symbols. The variance of the transmitted symbols $d_{n}$ and their soft estimates $\bar{d}_{n}$ are denoted $\sigma_{d}^{2}$ and $\sigma_{\bar{d}}^{2}$, respectively.

Exploiting the fact that $E_{X}\{X\}=E_{Y}\left\{E_{X I Y}\{X / Y\}\right\}$, we have

$$
E\left\{d_{n} \bar{d}_{k}^{*}\right\}=E\left\{E\left\{d_{n} \bar{d}_{k}^{*} / \mathbf{L}_{a}^{d e c}\right\}\right\}
$$

Accounting for definition (7) of $\bar{d}_{k}$ which is already conditioned to $\mathbf{L}_{a}^{\text {dec }}$, we obtain

$$
E\left\{d_{n} \bar{d}_{k}^{*}\right\}=E\left\{\bar{d}_{k}^{*} E\left\{d_{n} / \mathbf{L}_{a}^{d e c}\right\}\right\}
$$

Consequently, it follows from relation (7) that

$$
E\left\{d_{n} \bar{d}_{k}^{*}\right\}=E\left\{\bar{d}_{n} \bar{d}_{k}^{*}\right\}
$$

Since the soft estimate symbols $\bar{d}_{n}$ are assumed to be mutually independent owing to the presence of the bit interleaver in the turbo-equalization scheme, we have

$$
E\left\{d_{n} \bar{d}_{k}^{*}\right\}=\sigma_{\bar{d}}^{2} \delta_{n, k}
$$

Note that the parameter $\sigma_{\partial}^{2}$ is estimated in practice using (11). From (33) and (37), the variance $\sigma^{2}$ at the output of the equalizer is given by

$$
\sigma^{2}=\sigma_{d}^{2} \sum_{l \neq 0}\left|g_{l}\right|^{2}+\sigma_{d}^{2}\left(\sum_{l \neq 0}\left|q_{l}\right|^{2}-2 \Re e\left\{\sum_{l \neq 0} q_{l} g_{l}^{*}\right\}\right)+\sigma_{w}^{2} \sum_{l}\left|p_{l}\right|^{2}
$$


The last expression can be conveniently rewritten in the form

$$
\sigma^{2}=\left(\sigma_{d}^{2}-\sigma_{d}^{2}\right) \sum_{l \neq 0}\left|g_{l}\right|^{2}+\sigma_{d}^{2} \sum_{l \neq 0}\left|g_{l}-q_{l}\right|^{2}+\sigma_{w}^{2} \sum_{l}\left|p_{l}\right|^{2}
$$

From (15), $S N R_{\text {out }}$ is maximum when the conditional variance $\sigma^{2}$ is minimum. The r.h.s. of (39) is minimum when $\sigma_{d}^{2} \sum_{l \neq 0}\left|g_{l}-q_{l}\right|^{2}$ is minimum, yielding the following solution for $\tilde{Q}(v)$

$$
\tilde{Q}(v)=\tilde{G}(v)-g_{0}
$$

Note that the condition $q_{0}=0$ is respected. Substituting (40) into (39), we obtain

$$
\sigma^{2}=\left(\sigma_{d}^{2}-\sigma_{\bar{d}}^{2}\right) \sum_{l \neq 0}\left|g_{l}\right|^{2}+\sigma_{w}^{2} \sum_{l}\left|p_{l}\right|^{2}
$$

Consequently from (32) and (41), $S N R_{\text {out }}$ expression (15) becomes

$$
S N R_{\text {out }}=\frac{\left|g_{0}\right|^{2} \sigma_{d}^{2}}{\left(\sigma_{d}^{2}-\sigma_{\bar{d}}^{2}\right)\left(\sum_{l}\left|g_{l}\right|^{2}-\left|g_{0}\right|^{2}\right)+\sigma_{w}^{2} \sum_{l}\left|p_{l}\right|^{2}}
$$

Recalling definition (13) and applying Parseval's Theorem for discrete-time filters, we note that

$$
\begin{aligned}
& \sum_{l}\left|g_{l}\right|^{2}=T \int|\tilde{G}(v)|^{2} d v=T \int|\tilde{H}(v) \tilde{P}(v)|^{2} d v \\
& \sum_{l}\left|p_{l}\right|^{2}=T \int|\tilde{P}(v)|^{2} d v
\end{aligned}
$$

and

$$
\left|g_{0}\right|^{2}=\left|T \int \tilde{G}(v) d v\right|^{2}=\left|T \int \tilde{H}(v) \tilde{P}(v) d v\right|^{2}
$$

In order to simplify the mathematical expressions, we introduce the quantity $\tilde{F}(v)$ defined by the spectral factorization

$$
|\tilde{F}(v)|^{2}=\left(\sigma_{d}^{2}-\sigma_{\tilde{d}}^{2}\right)|\tilde{H}(v)|^{2}+\sigma_{w}^{2}
$$

Then substituting (43-45) into (42) yields

$$
S N R_{\text {out }}=\sigma_{d}^{2}\left(\frac{T \int|\tilde{P}(v) \tilde{F}(v)|^{2} d v}{\left|T \int \tilde{P}(v) \tilde{F}(v) \tilde{H}(v) / \tilde{F}(v) d v\right|^{2}}-\left(\sigma_{d}^{2}-\sigma_{\tilde{a}}^{2}\right)\right)^{-1}
$$


Using the Cauchy-Schwarz inequality, $S N R_{\text {out }}$ is bounded by

$$
S N R_{\text {out }} \leq \sigma_{d}^{2}\left(\frac{1}{T \int|\tilde{H}(v) / \tilde{F}(v)|^{2} d v}-\left(\sigma_{d}^{2}-\sigma_{\bar{d}}^{2}\right)\right)^{-1}
$$

with equality iff

$$
\tilde{P}(v)=\lambda \tilde{H}^{*}(v) /|\tilde{F}(v)|^{2}
$$

where $\lambda$ is a positive constant to be determined later. Let us define the value

$$
\beta=T \int|\tilde{H}(v)|^{2} /|\tilde{F}(v)|^{2} d v
$$

Then, $S N R_{\text {out }}$ can be expressed from (47) and (48) by

$$
S N R_{\text {out }}=\sigma_{d}^{2}\left(\beta^{-1}-\left(\sigma_{d}^{2}-\sigma_{\bar{d}}^{2}\right)\right)^{-1}
$$

From (13), (48) and (49), we have the following relation

$$
g_{0}=\lambda \beta
$$

From (32), the MSE (18) is given by

$$
M S E=E\left\{\left|\left(g_{0}-1\right) d_{n}\right|^{2}\right\}+\sigma^{2}
$$

and may be rewritten as a function of the parameter $\lambda$ using (50) and (15)

$$
M S E=|\lambda \beta|^{2}\left(\beta^{-1}-\left(\sigma_{d}^{2}-\sigma_{\bar{d}}^{2}\right)\right)+|\lambda \beta-1|^{2} \sigma_{d}^{2}
$$

Setting $\partial M S E / \partial \lambda=0$ results in

$$
\lambda=\sigma_{d}^{2} /\left(1+\beta \sigma_{a}^{2}\right)
$$

Finally, substituting (51) and (54) into (53), the MSE is given by

$$
M S E=\left(1-g_{0}\right) \sigma_{d}^{2}
$$

From (18), (32) and (55) it can be shown that the variance $\sigma^{2}$ is given by

$$
\sigma^{2}=g_{0}\left(1-g_{0}\right) \sigma_{d}^{2}
$$

which allows $S N R_{\text {out }}(15)$ to be expressed as

$$
S N R_{\text {out }}=g_{0} /\left(1-g_{0}\right)
$$




\section{REFERENCES}

[1] C. Douillard, A. Picart, P. Didier, M. Jézéquel, C. Berrou and A. Glavieux, "Iterative Correction of Intersymbol Interference : Turbo-Equalization", European Trans. Telecommun., Vol.6, no. 5, pp. 507-511, Sept./Oct. 1995.

[2] G. Bauch and V. Franz, "A Comparison of Soft-In / Soft-Out Algorithms for Turbo Detection," in Proc. IEEE ICT'98, pp. 259-263, Porto-Carras, Greece, June, 1998.

[3] W.H. Gerstacker and R. Schober, "Equalization Concept for EDGE," IEEE Trans. Wireless Commun., Vol. 1, no.1, pp. 190-199, Jan. 2002.

[4] A. O. Berthet, B. S. Ünal and R. Visoz, "Iterative Decoding of Convolutionally Encoded Signals Over Multipath Rayleigh Fading Channel," IEEE J. Select. Areas Commun., Vol. 19, no. 9, pp. 1729-1743, Sept. 2001.

[5] A. Duel-Hallen and C. Heegard, "Delayed Decision Feedback Sequence Estimation," IEEE Trans. Commun., Vol. 37, pp 428-436, May 1989.

[6] M.V. Eyuboglu and S.U. Qureshi, "Reduced-State Sequence Estimation with Set Partitionning and Decision Feedback," IEEE Trans. Commun., Vol. 36, pp. 13-20, Jan. 1988.

[7] G. Bauch and N. Al-Dhahir, "Iterative Equalization and Decoding with Channel Shortening Filters for Space-Time Coded Modulation," in Proc. IEEE VTC 2000, Vol. 4, pp. 1575-1582, 2000.

[8] B. Penther, D. Castelain and H. Kubo, "A Modified Turbo Detector for Long Delay Spread Channels," in Proc. $2^{\text {nd }}$ Int. Symp. turbo Codes \& Related Topics, pp. 295-298, Brest, France, Sept. 2000.

[9] A. Glavieux, C. Laot and J. Labat, "Turbo-Equalization over a Frequency Selective

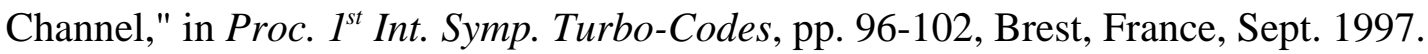

[10] C. Laot, A. Glavieux and J. Labat, "Turbo Equalization : Adaptive Equalization and Channel Decoding Jointly Optimized," IEEE J. Select. Areas Commun., Vol. 19, no. 9, pp. 1744-1752, Sept. 2001. 
[11] M. Tüchler, R. Koetter, A.C. Singer, "Turbo Equalization: Principles and New Results," IEEE Trans. Commun., Vol. 50, no. 5, pp. 754-767, May 2002.

[12] M. Tüchler, A.C. Singer and R. Koetter, "Minimum Mean Squared Error Equalization Using A Priori Information," IEEE Trans. Signal Processing, Vol. 50, no. 3, pp. 673-683, March 2002.

[13] X. Wang and V. Poor, "Iterative (Turbo) Soft Interference Cancellation and Decoding for Coded CDMA," IEEE Trans. Commun., Vol. 47, pp. 1046-1061, July 1999.

[14] A. Dejonghe and L. Vandendorpe, "Turbo-Equalization for Multilevel Modulation: an Efficient Low-Complexity Scheme," in Proc. IEEE ICC'02, pp. 1863-1867, 2002.

[15] A.M. Chan, G.W. Wornell, "A Class of Block-Iterative Equalizers for Intersymbols Interference Channels : Fixed Channels Results," IEEE Trans. Commun., Vol. 49, no. 11, pp. 1966-1976, Nov. 2001.

[16] D. Raphaeli and A. Saguy, "Reduced Complexity APP for Turbo Equalization," in Proc. IEEE ICC'02, pp. 1940-1943, 2002.

[17] C. Berrou and A. Glavieux, " Near Optimum Error Correcting Coding and Decoding : Turbo-codes," IEEE Trans. Commun. Vol. 44, no10, pp.1262-1271, Oct. 1996.

[18] J. Hagenaeur, "The Turbo principle : Tutorial Introduction and State of the Art," in Proc. $1^{\text {st }}$ Int. Symp. on Turbo-Codes, pp. 1-11, Brest, France, Sept. 1997

[19] S. Benedetto, D. Divsalar, G. Montorsi, F. Pollara, "Serial Concatenation of Interleaved Codes: Performance Analysis, Design, and Iterative Decoding," IEEE Trans. Inform. Theory, Vol. 44, pp. 909-926, May 1998.

[20] P. Robertson, E. Villebrun, and P. Hoeher, "A Comparison of Optimal and SubOptimal MAP Decoding Algorithms Operating in the Log Domain," in Proc IEEE ICC'95, pp. 1009-1013, Seattle, Washington, June 1995.

[21] S. Ten Brink, J. Speidel and R.H. Yan, "Iterative Demapping and Decoding for Multilevel Modulation," in Proc. IEEE GLOBECOM 1998, Vol. 1, pp. 579-584, 1998. 
[22] M. Witzke, S. Bäro, F. Schreckenback and J. Hagenauer, "Iterative Detection of MIMO Signals with Linear Detectors," in Proc. of Asilomar Conf. On Signals, Systems and Computers, ACSSC 2002, Pacific Grove, CA, Nov. 2002.

[23] S. M. Kay, Fundamentals of Signal Processing-Vol.1: Estimation Theory, Prentice Hall, 1993.

[24] S.U.H. Qureshi, "Adaptive Equalization," in Proc. IEEE, Vol. 73, pp. 1349-1387, Sept. 1985.

[25] A. Gersho and T.L. Lim, "Adaptive Cancellation of Intersymbol Interference for Data Transmission," Bell Syst.Tech. J., vol 60, no 11, pp. 1997-2021, Nov. 1981.

[26] M.S. Muller and J. Salz, "A Unified Theory of Data-Aided Equalization," Bell Syst.Tech. J., vol 60, no 9, pp. 2023-2038, Nov. 1981.

[27] R. Le Bidan, Turbo-Equalization for Bandwidth-Efficient Digital Communications over Frequency-Selective Channels, PhD. Thesis, INSA de Rennes, Rennes, France, Nov. 2003. [Online]. Available : http://www-sc.enst-bretagne.fr/ lebidan.

[28] M. Tüchler and J. Hagenauer, "Turbo Equalization Using Frequency Domain Equalizers, " in Proc. Of the $38^{\text {th }}$ Allerton Conf. On Comm., Control and Computing, Monticello, IL, USA, Oct. 2000.

[29] A. Fururskar, S. Mazur, F. Muller and H. Olofson, "EDGE, Enhanced Data Rates for GSM and TDMA/136 Evolution," IEEE Pers. Commun. Mag., pp.56-66, 1999.

[30] GSM 05.02: "Digital cellular telecommunications system (Phase 2+); Multiplexing and multiple access on the radio path".

[31] P. A. Laurent, "Exact and Approximate Construction of Digital Phase Modulations by Superposition of Amplitude Modulated Pulses (AMP)," IEEE Trans. Commun., Vol. 34, pp. 150-160, 1986.

[32] GSM 05.04: "Digital cellular telecommunications system (Phase 2+); modulation"

[33] GSM 05.05: "Digital cellular telecommunications system (Phase 2+); Radio transmission and reception".

[34] GSM 05.03: "Digital cellular telecommunications system (Phase 2+); Channel coding". 


\section{Figure captions}

Fig. 1. Discrete-time equivalent channel model

Fig. 2. A module of the turbo equalizer.

Fig. 3. Structure of the equalizer

Fig. 4. Modified structure of the equalizer

Fig. 5. Power distribution for the channels taps calculated with an oversampling factor of 36 and averaged over 1000 realizations of the GSM channel profiles.

Fig. 6. Comparison between the matrix- and FFT-based approaches for the computation of the linear equalizer in the EGPRS/MCS-8 context over the time-varying TU channel. Fig. 7. Performance of the turbo equalizer in the EGPRS/MCS-5 context over the timeinvariant ST channel.

Fig. 8. Performance of the turbo equalizer in the EGPRS/MCS-5 context over the timevarying (iFH) TU channel.

Fig. 9. Performance of the turbo equalizer in the EGPRS/MCS-5 context over the timevarying (iFH) HT and EQ channels.

Fig. 10. Performance of the turbo equalizer in the EGPRS/MCS-8 context over the time-invariant ST channel and the time-varying (iFH) TU channel.

Fig. 11. Performance of the turbo equalizer in the EGPRS/MCS-9 context over timeinvariant ST channel and the time-varying (iFH) TU channel. 


\section{Figures}

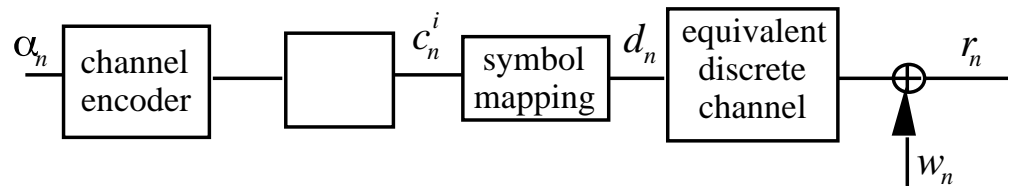

Fig.1. Discrete-time equivalent channel model

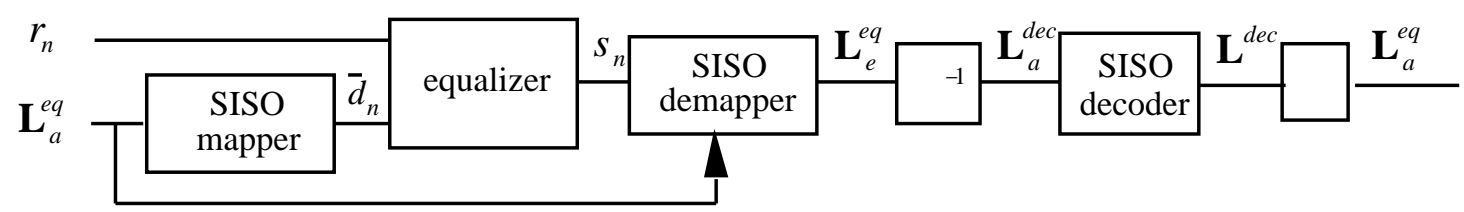

Fig. 2. A module of the turbo equalizer.

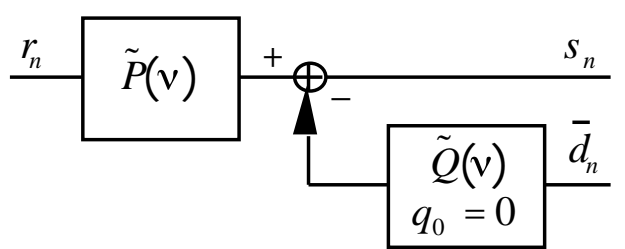

Fig. 3. Structure of the equalizer

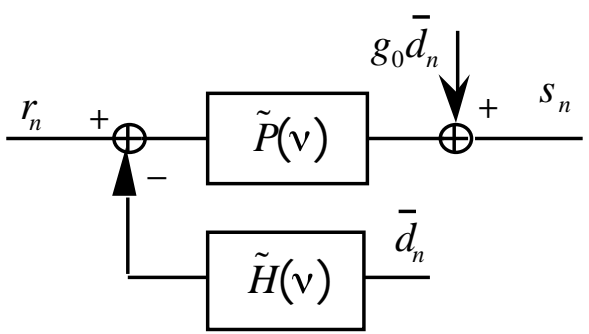

Fig. 4. Modified structure of the equalizer 

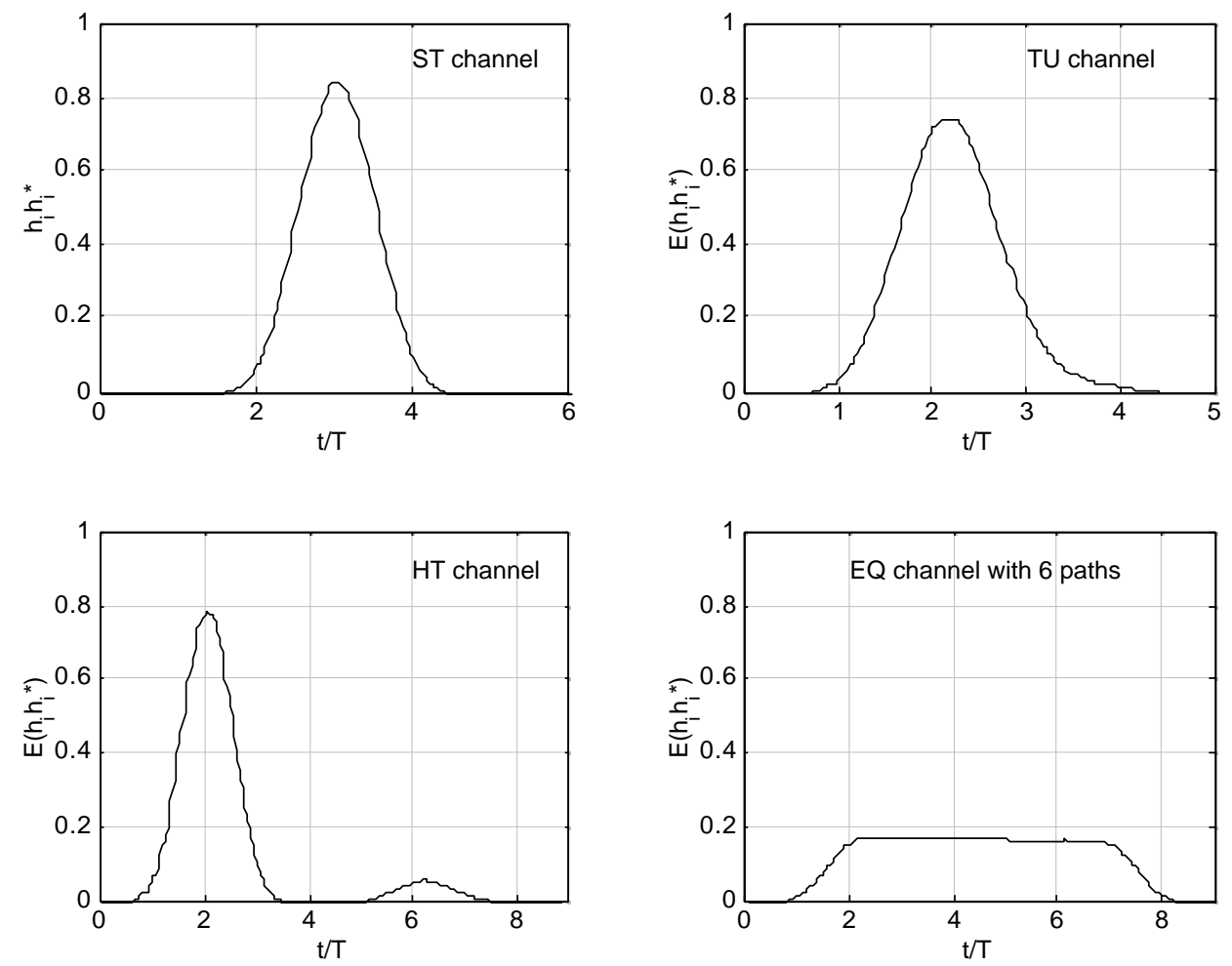

Fig. 5. Power distribution for the channels taps calculated with an oversampling factor of 36 and averaged over 1000 realizations of the GSM channel profiles. 


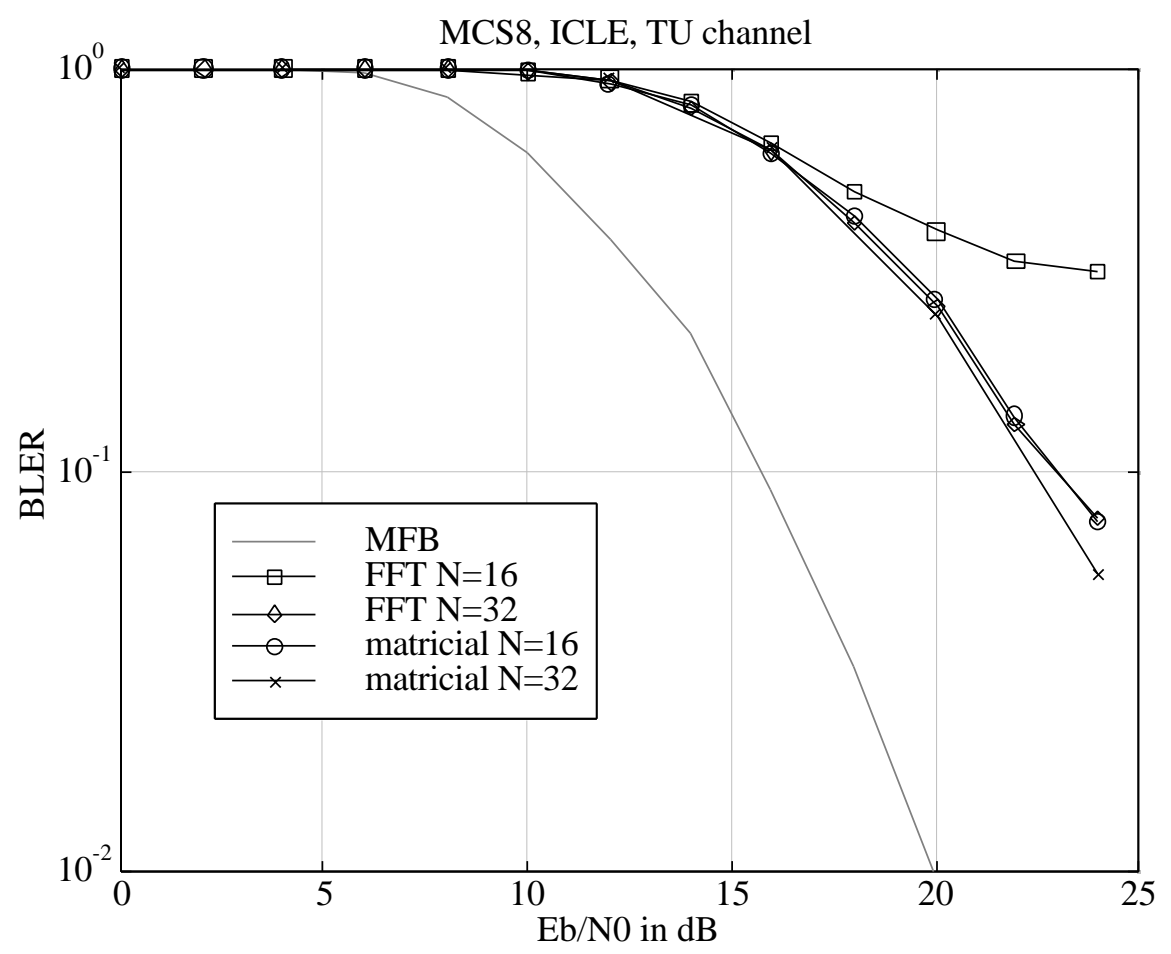

Fig. 6. Comparison between the matrix- and FFT-based approaches for the computation of the linear equalizer in the EGPRS/MCS- 8 context over the time-varying TU channel.
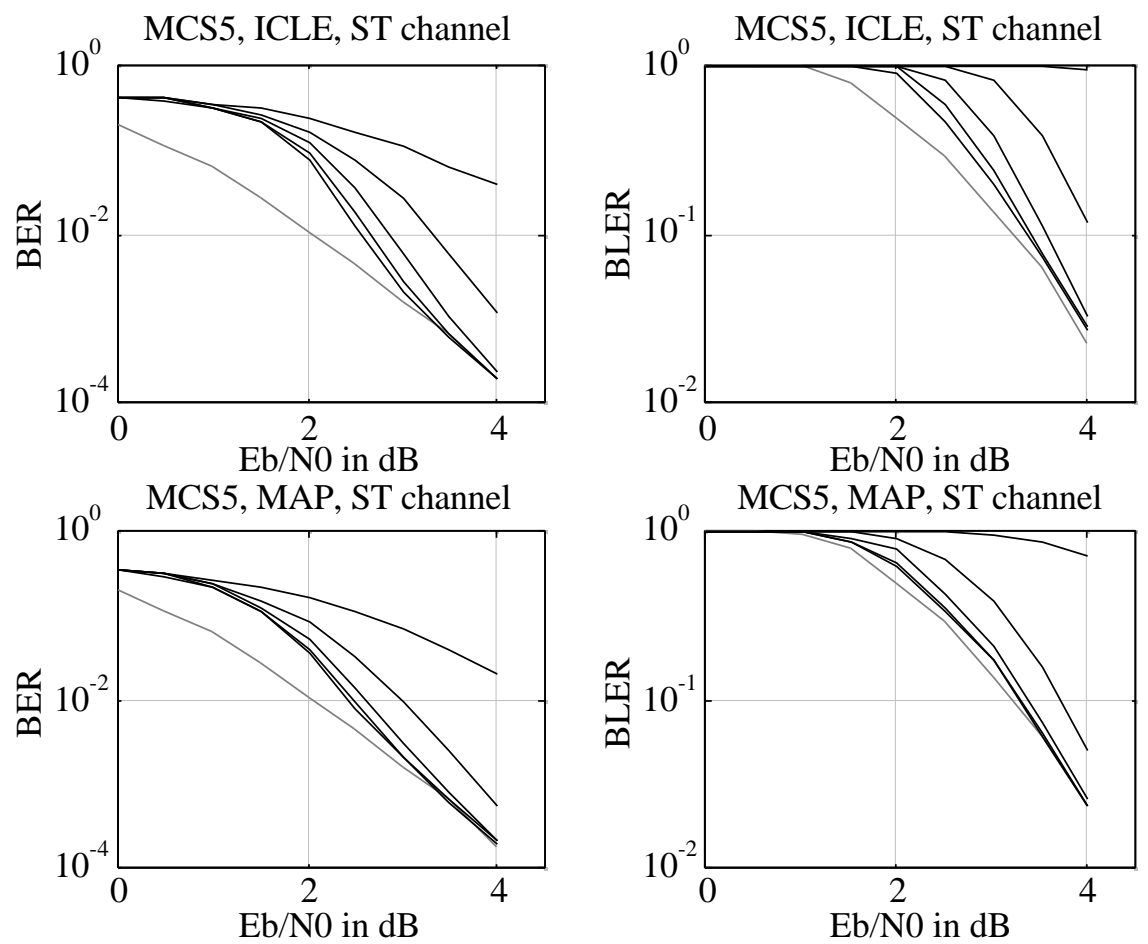

Fig. 7. Performance of the turbo equalizer in the EGPRS/MCS-5 context over the time-invariant ST channel. 

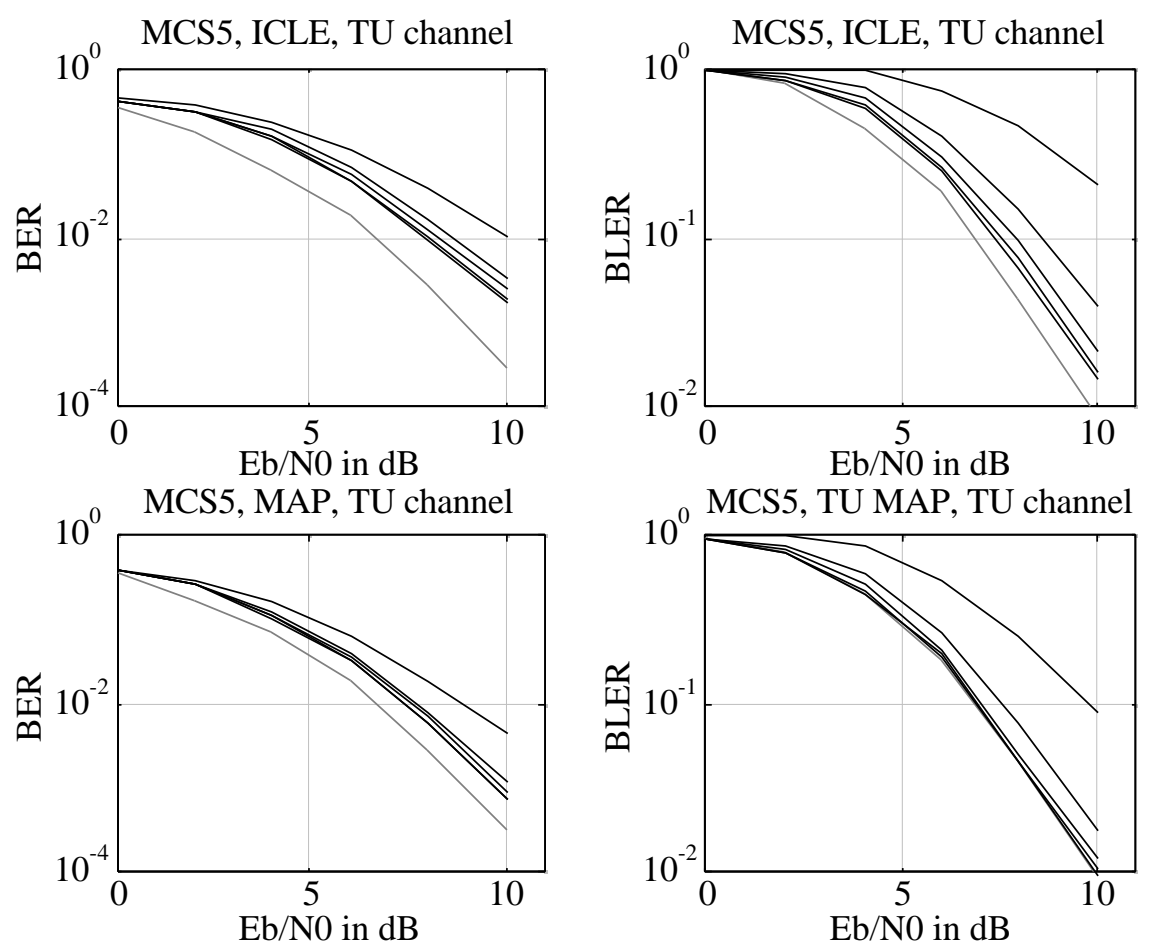

Fig. 8. Performance of the turbo equalizer in the EGPRS/MCS-5 context over the time-varying (iFH) TU channel.
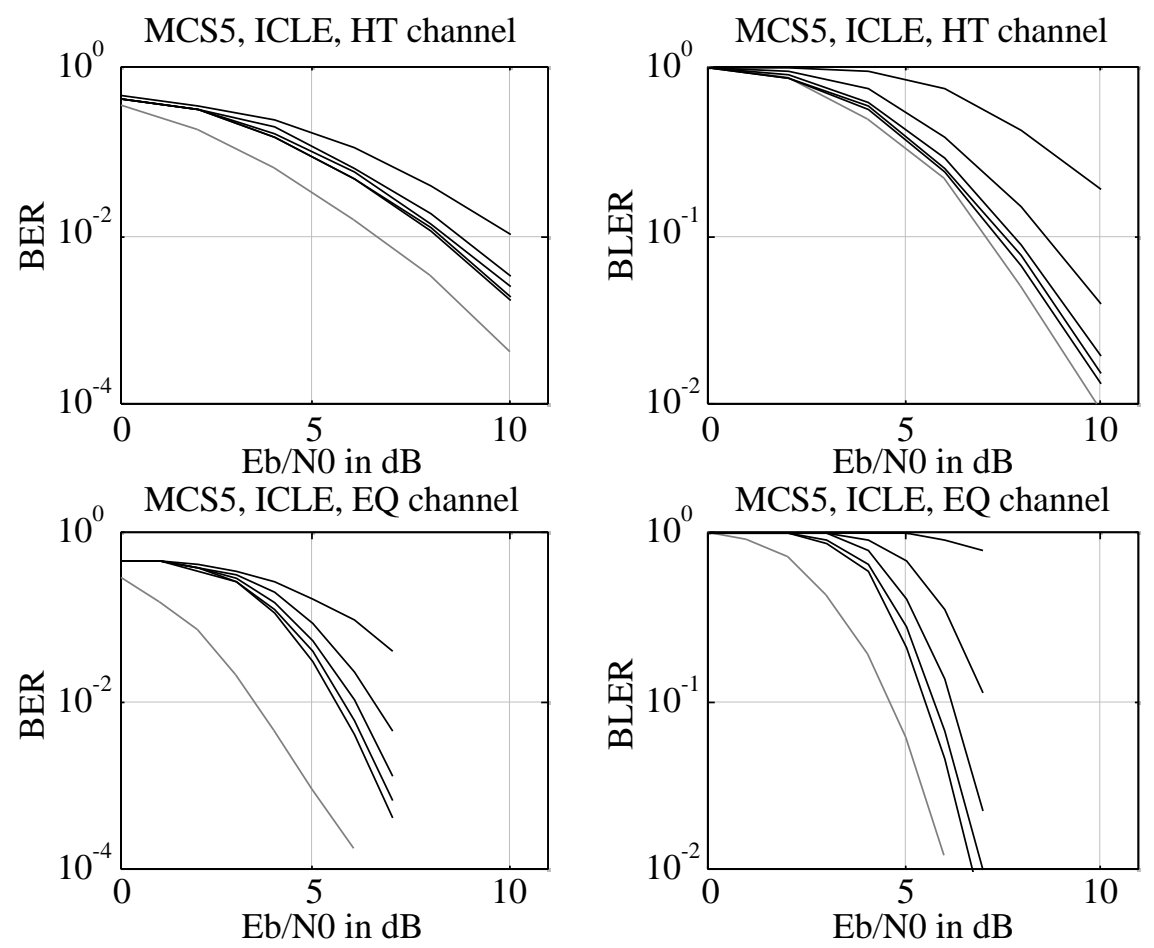

Fig. 9. Performance of the turbo equalizer in the EGPRS/MCS-5 context over the time-varying (iFH) HT and EQ channels. 

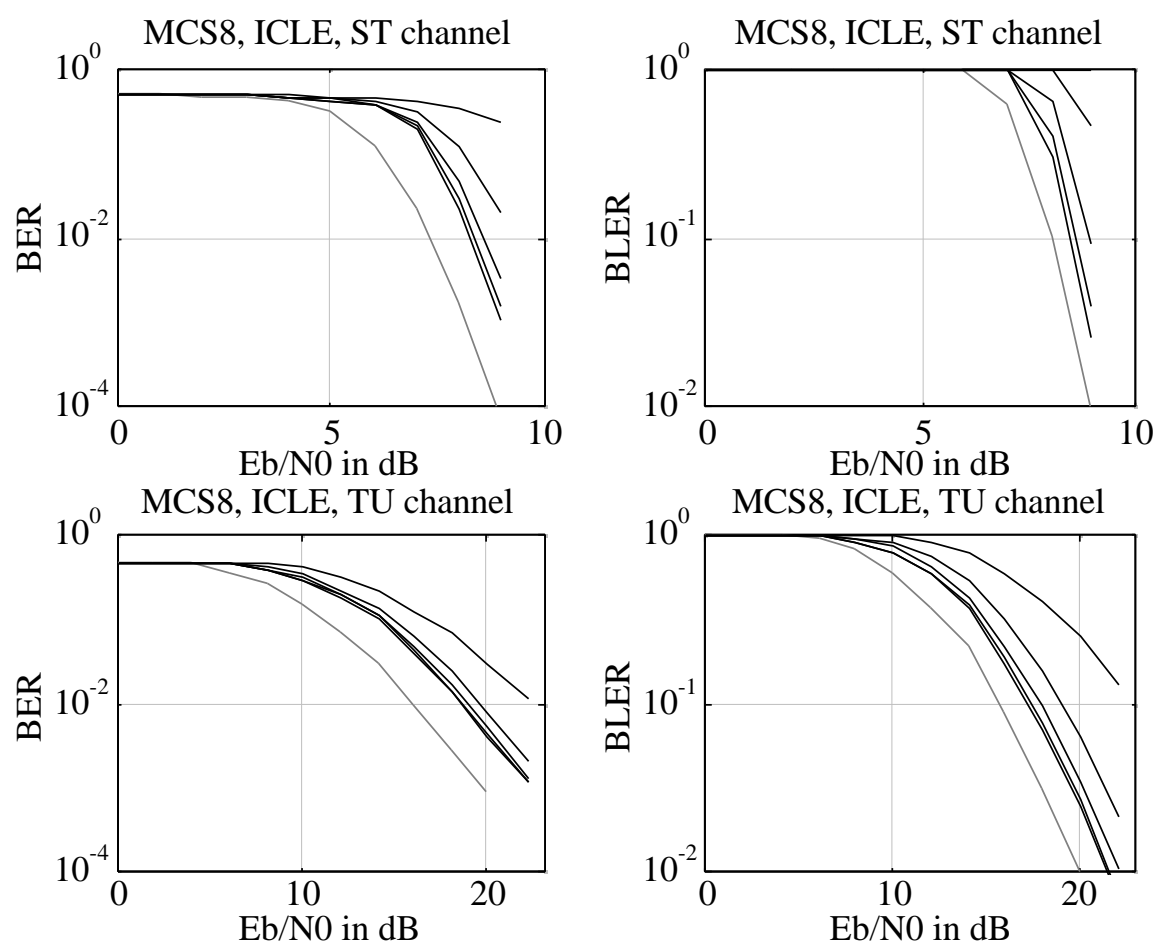

Fig. 10. Performance of the turbo equalizer in the EGPRS/MCS-8 context over the time-invariant ST channel and the time-varying (iFH) TU channel.
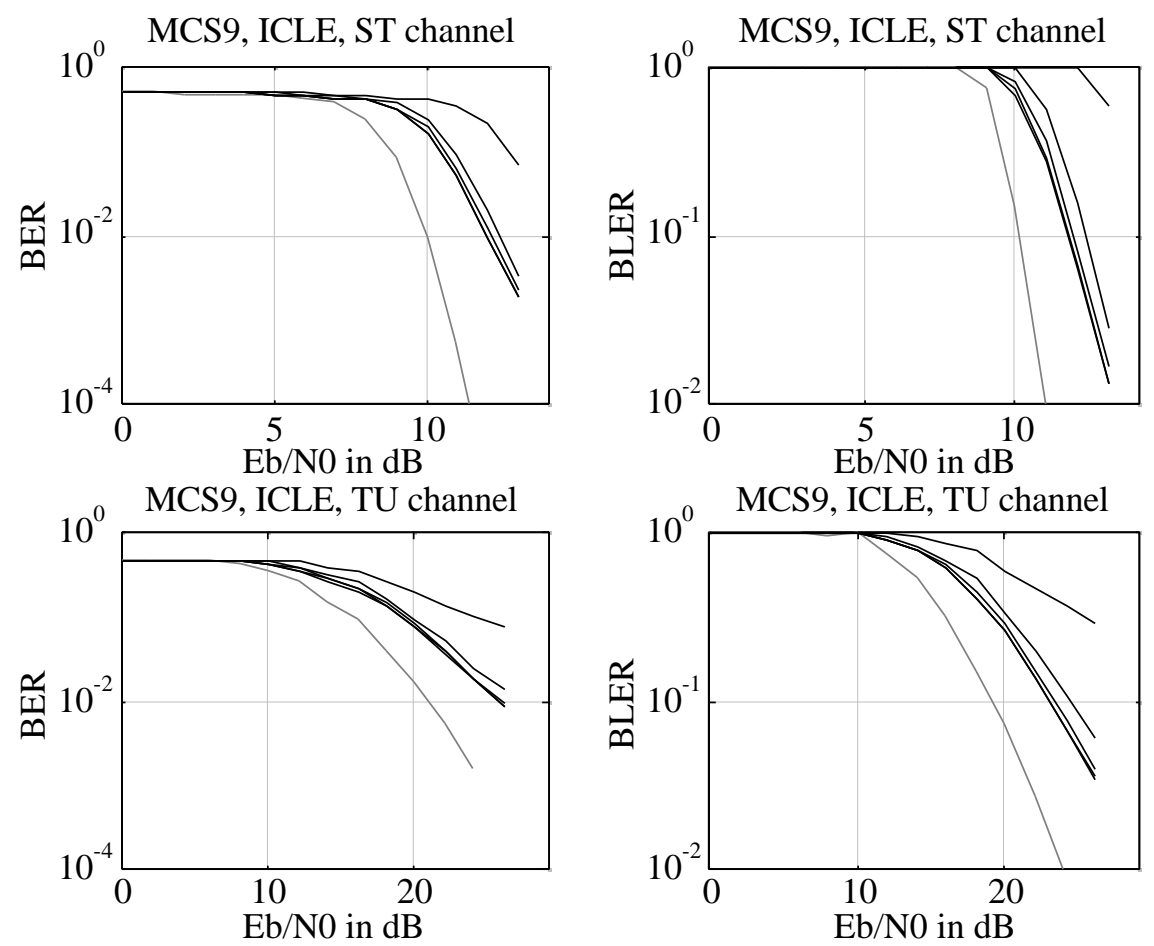

Fig. 11. Performance of the turbo equalizer in the EGPRS/MCS-9 context over the time-invariant ST channel and the time-varying (iFH) TU channel. 